\begin{tabular}{ll|l} 
Case Reports in & \multicolumn{2}{c}{ Case Rep Gastroenterol 2020;14:242-247 } \\
\cline { 2 - 3 } Gastroenterology & $\begin{array}{l}\text { DOl: 10.1159/000507014 } 2020 \text { The Author(s) } \\
\text { Published online: April 29, } 2020\end{array}$ & $\begin{array}{l}\text { Kablished by S. Karger AG, Basel } \\
\text { www.karger.com/crg }\end{array}$ \\
\cline { 2 - 3 } & $\begin{array}{l}\text { This article is licensed under the Creative Commons Attribution-NonCommercial } 4.0 \\
\text { International License (CC BY-NC) (http://www.karger.com/Services/OpenAccessLicense). } \\
\text { Usage and distribution for commercial purposes requires written permission. }\end{array}$
\end{tabular}

\title{
Amoxicillin-Clavulanate-Induced Ischaemic Colitis
}

\author{
Marco Alonge $^{a} \quad$ Federica Benini $^{a} \quad$ Rosanna Cannatelli ${ }^{b}$ \\ Alessandro Pozzi ${ }^{a}$ Guido Missale ${ }^{c}$ Vincenzo Villanaccid ${ }^{d}$ Chiara Ricci $^{a}$ \\ ${ }^{a}$ Gastroenterology Unit, Department of Clinical and Experimental Sciences, University of \\ Brescia and Spedali Civili, Brescia, Italy; ${ }^{b}$ Gastroenterology Unit, Department of Clinical \\ and Experimental Sciences, University of Milan and Spedali Civili, Brescia, Italy; ${ }^{\mathrm{C} D i g e s t i v e}$ \\ Endoscopy Unit, Department of Clinical and Experimental Sciences, University of Brescia \\ and Spedali Civili, Brescia, Italy; dPathological Anatomy Division, Department of Molecular \\ and Translational Medicine, University of Brescia and Spedali Civili, Brescia, Italy
}

\section{Keywords}

Ischaemic colitis · Amoxicillin-clavulanate · Pseudomembranes · Pseudomembranous ischaemic colitis

\begin{abstract}
Ischaemic colitis (IC) is the most frequent form of ischaemia of the digestive tract. Due to the worldwide increasing use of medications, there is a growing interest in drug-induced IC. This study reports a rare case of IC directly due to amoxicillin-clavulanate intake. The objective of the study was to describe the evolution of this novel manifestation. An 18-year-old man, nonsmoker, with an insignificant medical history, presented with diarrhoea and cramping abdominal pain that started the day following the end of a 10-day amoxicillin-clavulanate course for recent upper respiratory tract infection. Stool cultures including Clostridium difficile toxin testing were negative. Colonoscopy documented an erosive-ulcerative colitis of the sigmoid and the descending colon. Histological examination of the colon biopsies revealed an IC with focal pseudomembranous areas in the descending-sigmoid colon. Thrombophilia screening tests were negative. The patient was discharged from the hospital without symptoms, and another colonoscopy was performed 3 weeks after the previous one, which documented normal endoscopic and histological findings. Amoxicillin-clavulanate IC is a very rare condition and should be suspected once infectious diseases, vascular/haemodynamic causes and a
\end{abstract}




\section{Case Reports in Gastroenterology}

Case Rep Gastroenterol 2020;14:242-247 DOI: 10.1159/000507014

(c) 2020 The Author(s). Published by S. Karger AG, Basel www.karger.com/crg

Alonge et al.: Amoxicillin-Clavulanate-Induced Ischaemic Colitis

prothrombotic/hypercoagulable state have been excluded. Immediate discontinuation of the antibiotic leads to rapid disease remission.

\section{Introduction}

Ischaemic colitis (IC) is the most frequent form of ischaemia of the digestive tract, even if it is often misdiagnosed because of its temporary, mild clinical manifestation and the similarity to other diseases such as infectious colitis or inflammatory bowel disease. The incidence of IC has considerably risen over the years: from 6.1 cases/100,000 person-years in 1976-1980 to $22.9 / 100,000$ in 2005-2009 [1]. Its prevalence increases with age and comorbidity [1]. It is usually difficult to determine the precise site of the ischaemic insult; however, we should suspect IC in elderly patients with small vessel disease and various comorbidities. Most of cases occur in patients over 60 years, although younger patients may also be affected [2].

A lot of conditions may induce IC: arterial emboli, thrombosis or trauma [3]; hypoperfusion states and shock due to a variety of causes such as hypovolaemia or sepsis [4]; and mechanical colonic obstruction due to tumours, adhesions, volvuli, hernias, diverticulitis or prolapse [4]. Drug-induced IC is also a well-represented entity. Several classes of pharmacologic agents are associated with IC [5]: appetite suppressants like phentermine, chemotherapeutic agents like vinca alkaloids and taxanes, constipation-inducing medications, decongestants like pseudoephedrine, cardiac glucosides, diuretics, ergot alkaloids, hormonal therapies, statins, illicit drugs, immunosuppressive agents, laxatives, non-steroidal anti-inflammatory drugs, psychotropic medications, serotonin agonists/antagonists, vasopressors and antibiotics. IC is a rising condition among drug-induced adverse effects and diseases.

This study reports a rare case of pseudomembranous IC directly due to amoxicillin-clavulanate intake. Pseudomembranes, seen on histological examination, represented an early manifestation of IC. The objectives of the study were to describe the evolution of this novel manifestation. Even if more studies on this issue are required, we documented complete clinical and histological remission after antibiotic discontinuation. There is only 1 case of amoxicillin-related IC in the literature, but it is described as an effect of anaphylactic shock due to antibiotic allergy [6].

\section{Case Report}

This is a report on an 18-year-old man with an insignificant past medical history, nonsmoker, and with recent upper respiratory tract infection, treated with a course of antibiotics. One amoxicillin-clavulanate 875/125 mg film-coated tablet had been administered orally twice daily for 10 days, according to the general practitioner's prescription. During the night following the final day of antibiotic course, the patient developed diarrhoea with cramping and stabbing abdominal pain, mostly localised in the lower abdominal quadrants. The following days, the patient experienced passing of frequent watery stools, about 15 times a day, with bowel movement-induced abdominal pain, in the absence of nausea, fever or vomiting. For this reason, the patient decided to go to the hospital. He referred regular intestinal transit, with no abdominal distress or discomfort, in the past years, but also a family history of colorectal cancer and Crohn's disease. The patient denied eating unsafe food, recent trips abroad or non-steroidal anti-inflammatory drug intake. 
The standard haematologic tests and blood chemistry tests showed leucocytosis (WBC $13,520 / \mu \mathrm{L}$ ), a slight increase in C-reactive protein (CRP) levels (CRP $9.2 \mathrm{mg} / \mathrm{L}$ ) and hypokalaemia $\left(\mathrm{K}^{+} 3.1 \mathrm{mmol} / \mathrm{L}\right)$. We then performed a colonoscopy, which documented erosive-ulcerative colitis of the sigmoid and the descending colon (Fig. 1), with a normal rectal mucosal surface. Histological examination of the colon biopsies revealed a normal rectal mucosa with prolapsed areas and an IC appearance (coagulative necrosis of the superficial epithelium) with focal pseudomembranous areas (aggregates of hyaline material intermingled with eosinophils) in the descending-sigmoid colon (Fig. 2). During hospitalisation, the laboratory tests showed a rapid decrease in CRP (CRP $<2.90 \mathrm{mg} / \mathrm{L}$ ) and leucocyte count (WBC 4,550/ $\mu \mathrm{L}$ ), as well as clinical remission of symptoms like diarrhoea and abdominal pain. Stool cultures, including Clostridium difficile (CD) toxin testing, were negative. Thrombophilia screening tests (protein C, protein S, lupus anticoagulant, D-dimer test, factor II mutation, factor V mutation and anti-cardiolipin antibodies) were also performed to exclude a prothrombotic/hypercoagulable state, with negative results.

The patient was asymptomatic when discharged from the hospital. Colonoscopy performed 3 weeks after antibiotic discontinuation did not reveal any pathological finding. The colonic luminal surface seen during the colonoscopy and the histological examination of the biopsies were both totally normal.

\section{Discussion}

Beta-lactams represent the most commonly prescribed antibiotic class and are often associated with various adverse manifestations: immunoglobulin E-mediated type I reactions, serum sickness, and dermatologic, neurologic, pulmonary, hepatobiliary, renal, haematologic and gastrointestinal reactions. Diarrhoea is a common complication of antibiotic therapy, most frequently caused by oral antibiotics such as ampicillin or amoxicillin [7]. All antibiotics can predispose to CD colitis, especially ampicillin among the class of beta-lactams [8].

Among amoxicillin-clavulanate-related reactions, diarrhoea is the most frequent manifestation (3-34\% of cases) and its incidence varies depending on the dose and regimen used. A very small percentage $(<1 \%)$ of adverse manifestations are presented in post-marketing and case reports. Only 1 case of IC secondary to amoxicillin-clavulanate has been reported in the literature, even if caused by the hypotensive state suffered during the anaphylactic episode induced by antibiotic intake [6].

It is well known that the spectrum of clinical IC manifestations is heterogeneous, including mild and reversible forms, acute forms like pseudomembranous colitis (PMC), chronic diseases and fulminant pancolitis. The same heterogeneity is seen on histological examination, and the diverse microscopic aspects are surely influenced by the severity of ischaemic damage and by the time elapsed since the initial injury. Pseudopolyps are a direct effect of submucosal injury, whereas pseudomembranes occur subsequently, when rapid mucosal reperfusion leads to a localised inflammatory response. The pseudomembranes on the mucosa are formed by expulsion of inflammatory infiltrate from the lamina propria onto the luminal surface. PMC tends to be usually associated with CD infection, but it is also associated and caused by ischaemia. Ischaemia-related PMC is rarer and more difficult to diagnose, because pseudomembranes are mostly seen in the early phases of the ischaemic process [9]. A prospective, multicentre study on 364 patients diagnosed with IC showed ulcers with pseudomembranes in $13.1 \%$ of the endoscopic biopsies and $24 \%$ of the surgical biopsies; this finding was statistically significantly more common in the first $48 \mathrm{~h}$ of presentation [9]. 
According to these data, in our case the colonoscopy with biopsies was performed within the first $48 \mathrm{~h}$ after symptom onset, and the pseudomembranes were detected only by histological microscopic examination. In this case, the relationship between antibiotic intake and symptom onset helped us to suspect an adverse drug reaction. Given the recent medical history of the patient, our suspicion was an adverse reaction to amoxicillin-clavulanate, which was the only drug administered immediately before the IC outbreak; the antibiotic may have triggered a local vascular reaction that damaged the colonic mucosa. After excluding any smoking habit, which is a well-known risk factor for IC, thrombophilia screening tests were performed to be sure that no prothrombotic state underlay the ischaemic condition of the colitis. In addition, the sudden remission from the symptoms with a rapid decrease in inflammatory indexes as well as the histological report let us exclude an onset of inflammatory bowel disease. The two aspects confirming the hypothesis of a drug-related condition were the histological finding of a pseudomembranous IC (CD negative) and the complete endoscopic remission after a brief period of 3 weeks since amoxicillin-clavulanate withdrawal. In this specific case, the IC went rapidly into remission with antibiotic discontinuation; the pseudomembranes seen only on microscopic examination represented an early marker of IC.

In conclusion, IC is a widely heterogeneous disease which may highlight vascular or haemodynamic problems. Once these causes have been assessed and excluded, drug-induced IC should be considered. Amoxicillin-clavulanate may cause antibiotic-induced IC, which, according to our experience, tends to be self-limiting after drug discontinuation. The described clinical report underlines the potential unusual side effects of antibiotics routinely used; prompt withdrawal of the medication may limit significant disease morbidity and mortality.

\section{Acknowledgments}

The authors received no financial support for the research, authorship and/or publication of this article.

\section{Statement of Ethics}

All procedures were performed in accordance with the ethical standards laid down in the 1964 Declaration of Helsinki and its later amendments. Written informed consent for publication of the clinical details and clinical images was obtained from the patient. A copy of the consent form is available for review by the editor of this journal.

\section{Disclosure Statement}

The authors have no conflicts of interest to declare.

\section{Funding Sources}

This case report received no funding support for its preparation. 


\section{Author Contributions}

M.A.: literature research, writing and editing the manuscript; F.B.: clinical management of the patient, revision and final approval of the manuscript; A.P., G.M. and V.V.: revision and final approval of the manuscript; C.R.: revision and critical review and approval of the manuscript. All authors read and approved the final manuscript.

\section{References}

1 Yadav S, Dave M, Edakkanambeth Varayil J, Harmsen WS, Tremaine WJ, Zinsmeister AR, et al. A populationbased study of incidence, risk factors, clinical spectrum, and outcomes of ischemic colitis. Clin Gastroenterol Hepatol. 2015 Apr;13(4):731-8.e1.

2 Binns JC, Isaacson P. Age-related changes in the colonic blood supply: their relevance to ischaemic colitis. Gut. 1978 May;19(5):384-90.

3 Gandhi SK, Hanson MM, Vernava AM, Kaminski DL, Longo WE. Ischemic colitis. Dis Colon Rectum. 1996 Jan;39(1):88-100.

4 Green BT, Tendler DA. Ischemic colitis: a clinical review. South Med J. 2005 Feb;98(2):217-22.

5 Hass DJ, Kozuch P, Brandt LJ. Pharmacologically mediated colon ischemia. Am J Gastroenterol. 2007 Aug;102(8):1765-80.

6 Pérez-Carral C, Carreira J, Vidal C. Acute ischaemic colitis due to hypotension and amoxicillin allergy. Postgrad Med J. 2004 May;80(943):298-9.

7 Gillies M, Ranakusuma A, Hoffmann T, Thorning S, McGuire T, Glasziou P, et al. Common harms from amoxicillin: a systematic review and meta-analysis of randomized placebo-controlled trials for any indication. CMAJ. 2015 Jan;187(1):E21-31.

8 Kelly CP, Pothoulakis C, LaMont JT. Clostridium difficile colitis. N Engl J Med. 1994 Jan;330(4):257-62.

9 Montoro MA, Brandt LJ, Santolaria S, Gomollon F, Sánchez Puértolas B, Vera J, et al.; Workgroup for the Study of Ischaemic Colitis of the Spanish Gastroenterological Association (GTECIE-AEG). Clinical patterns and outcomes of ischaemic colitis: results of the Working Group for the Study of Ischaemic Colitis in Spain (CIE study). Scand J Gastroenterol. 2011 Feb;46(2):236-46. 


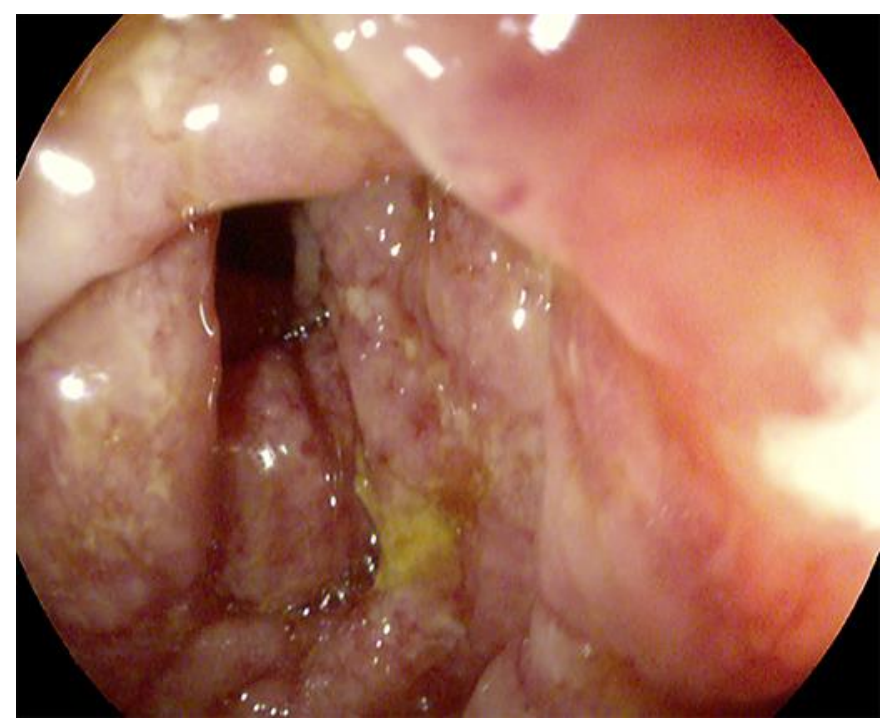

Fig. 1. Colonoscopy image showing an oedematous and hyperaemic mucosa of the sigmoid tract with multiple erosions and ulcerations, tending to be confluent and circumferential.

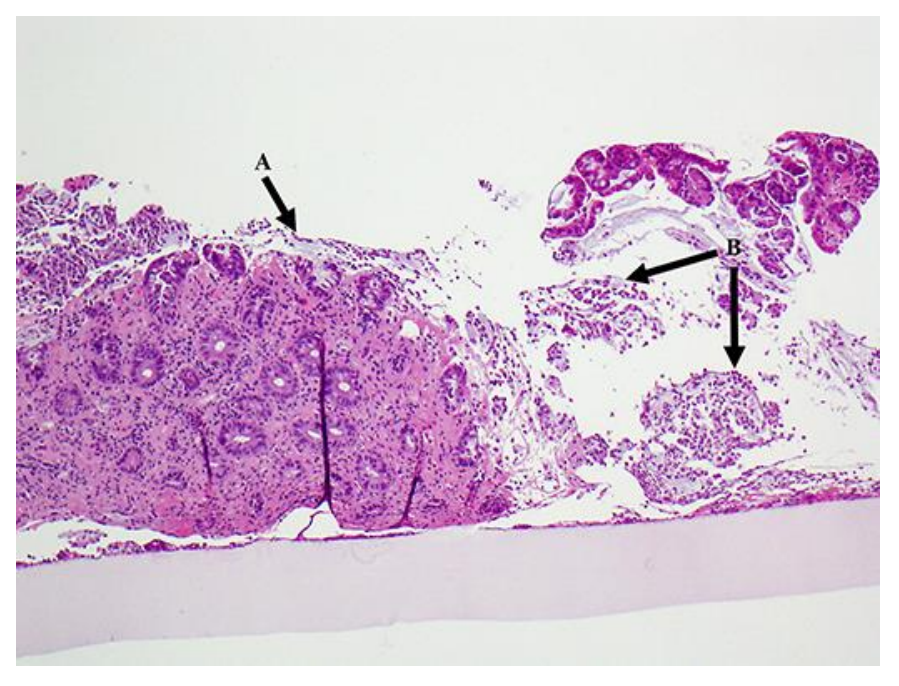

Fig. 2. Histological examination of the descending-sigmoid colon biopsies showed ischaemic colitis (A, arrow) with focal pseudomembranous areas (B, arrows): highly hyperplastic glandular elements mixed with atrophic crypt elements immersed in a homogeneously inflamed and vascularised lamina propria with erosion of the surface's coat, in which there were mixed inflammatory elements like neutrophils and most of all eosinophils. HE. $\times 20$. 